\title{
Michel Décorps
}

\section{Imagerie de résonance magnétique Bases physiques et méthodes}

\author{
S A V O I R S A C T U E L S \\ EDP Sciences/CNRS Éditions
}


Illustration de couverture: Coupe sagittale, contraste T1, technique SENSE. Image plateforme IRM de Grenoble (SFR1).

Imprimé en France.

(C) 2011, EDP Sciences, 17, avenue du Hoggar, BP 112, Parc d'activités de Courtabœuf, 91944 Les Ulis Cedex A

et

CNRS Éditions, 15, rue Malebranche, 75005 Paris.

Tous droits de traduction, d'adaptation et de reproduction par tous procédés réservés pour tous pays. Toute reproduction ou représentation intégrale ou partielle, par quelque procédé que ce soit, des pages publiées dans le présent ouvrage, faite sans l'autorisation de l'éditeur est illicite et constitue une contrefaçon. Seules sont autorisées, d'une part, les reproductions strictement réservées à l'usage privé du copiste et non destinées à une utilisation collective, et d'autre part, les courtes citations justifiées par le caractère scientifique ou d'information de l'œuvre dans laquelle elles sont incorporées (art. L. 122-4, L. 122-5 et L. 335-2 du Code de la propriété intellectuelle). Des photocopies payantes peuvent être réalisées avec l'accord de l'éditeur. S'adresser au : Centre français d'exploitation du droit de copie, 3, rue Hautefeuille, 75006 Paris. Tél. : 0143269535.

ISBN EDP Sciences 978-2-7598-0000-1

ISBN CNRS ÉDITIONS 978-2-271-07233-7 